PROCEEDINGS OF THE

AMERICAN MATHEMATICAL SOCIETY

Volume 29, Number 3, August 1971

\title{
THE ITERATES OF A CONTRACTION AND ITS ADJOINT ${ }^{1}$
}

\author{
JOHN A. R. HOLBROOK
}

ABstract. We prove that when $T$ is a contraction on Hilbert space the size of lim sup $\left|\left(\left(T^{*}\right)^{n} h, g\right)\right|$ is controlled by that of lim sup $\left|\left(T^{n} h, g\right)\right|$. We give an application to Fourier-Stieltjes coefficients. Important in the proof is a generalization of the technique of orthogonal projection.

1. Introduction. Our purpose is to prove the following theorem.

THEOREM 1. Given any $\epsilon>0$, there is a $\delta>0$ such that, whenever $T$ is a contraction on a Hilbert space $\mathfrak{H C}$ and $h, g \in \mathfrak{H C}$ satisfy $\|h\|,\|g\| \leqq 1$,

$$
\limsup _{n \rightarrow \infty}\left|\left(T^{n} h, g\right)\right| \leqq \delta \Rightarrow \limsup _{n \rightarrow \infty}\left|\left(\left(T^{*}\right)^{n} h, g\right)\right| \leqq \epsilon .
$$

In fact, we can (assuming $\epsilon \leqq 1$ ) take $\delta=\left(\epsilon^{2} / 4\right) \exp \left(-2 \epsilon^{-2}\right)$.

It is easy to see that this theorem implies the following result on Fourier-Stieltjes coefficients, proved recently by de Leeuw and Katznelson (see [6]).

THEOREM 2. Given any $\epsilon>0$, there is a $\delta>0$ such that, for any complex Borel measure $\mu$ on $[0,2 \pi)$ with $\|\mu\| \leqq 1$,

$$
\limsup _{n \rightarrow \infty}|\hat{\mu}(n)| \leqq \delta \Rightarrow \limsup _{n \rightarrow \infty}|\hat{\mu}(-n)| \leqq \epsilon .
$$

Theorem 2 is a quantitative generalization of the following old result of Rajchman (proved, in essence, in Zygmund [5, Chapter XII, $\S 10])$.

THEOREM 3. If $\mu$ is a complex Borel measure on $[0,2 \pi)$, then $\hat{\mu}(n) \rightarrow 0 \Leftrightarrow \hat{\mu}(-n) \rightarrow 0$.

We state also the corresponding result for operators, which follows directly from Theorem 1.

THEOREM 4. If $T$ is a contraction on $\mathfrak{F}$, then, for any $h, g \in \mathfrak{F}$,

$$
\left(T^{n} h, g\right) \rightarrow 0 \Leftrightarrow\left(\left(T^{*}\right)^{n} h, g\right) \rightarrow 0 .
$$

Received by the editors May 26, 1970 and, in revised form, November 20, 1970. AMS 1969 subject classifications. Primary 4710, 4750, 4615; Secondary 4210.

Key words and phrases. Contraction operator, Fourier-Stieltjes coefficients, orthogonal projection.

1 Work supported in part by AF Grant AFOSR 1322-67.

Copyright (c) 1971, American Mathematical Society 
Finally, we point out that Theorem 4 is a more precise, pointwise version of the following theorem of Foguel (see [1]).

ThEOREM 5. If $T$ is a contraction on $\mathfrak{H C}$ and $h \in \mathcal{H}$, then $T^{n} h \rightarrow 0$ weakly $\Leftrightarrow\left(T^{*}\right)^{n} h \rightarrow 0$ weakly.

We remark that one of the interesting applications of Theorem 5 is to the construction of power-bounded operators which are not similar to any contraction (see Foguel [2] or Halmos [4]).

By means of the Sz.-Nagy theory of "strong unitary dilations" (see, for example, Sz.-Nagy and Foias,, [3, Chapter I, 4]), we can write the quantities $\left(T^{n} h, g\right)$ and $\left(\left(T^{*}\right)^{n} h, g\right)$ occurring in Theorem 1 in the form $\left(U^{ \pm n} h, g\right)$, where $U$ is a unitary operator (on some Hilbert space $\mathfrak{K}$ containing $\mathfrak{F}$ ). By the spectral theory for unitary operators, we then have $\left(U^{ \pm n} h, g\right)=\hat{\mu}( \pm n)$, for a certain complex measure $\mu$ on $[0,2 \pi)$. It is not hard to see that, by this means, we can recover Theorem 1 (leaving aside the estimate for $\delta$ ) from Theorem 2. The point here is that we obtain directly the more general operator formulation (Theorem 1) by techniques furnishing an interesting alternative to the arguments of de Leeuw and Katznelson in [6]. Our proof, given in $\$ 2$, is mainly geometric in nature and depends upon a quantitative generalization of the method of orthogonal projection (see Lemma 3). If one simply wants to prove Theorem 4 , the argument can be greatly simplified; in particular one needs only the elementary form of orthogonal projection (that is, projection onto subspaces).

Finally we remark that measures constructed by de Leeuw and Katznelson (see $[6, \S 4]$ ) show that, in general, we cannot take $\delta=\epsilon$ in Theorems 1 and 2; the exact nature of the dependence of $\delta$ on $\epsilon$ seems to be unknown.

2. Proof of Theorem 1. We first prepare some lemmas.

LEMMA 1. If $S$ is a contraction on $\mathfrak{H}$ and $a, b \in \mathfrak{H C}$, then

$$
\left(S^{n} a, b\right)-\left(S^{n+1} a, S b\right) \underset{n}{\rightarrow} 0 .
$$

Proof. As Foguel has pointed out (see $[1$, p. 551, 1a $]$ ), $\left\|S^{n} a-S^{*} S^{n+1} a\right\|^{2} \leqq\left\|S^{n} a\right\|^{2}-\left\|S^{n+1} a\right\|^{2}$ whenever $\left\|S^{*}\right\| \leqq 1$. But $\left\|S^{n} a\right\|$ is a nonincreasing, therefore convergent, sequence.

We shall also need the following inequality of Foguel (see [1]), which we prove directly from Lemma 1.

Lemma 2. If $S$ is a contraction on $\mathfrak{H}$ and $a, b \in \mathfrak{H}$, then

$$
\lim \sup \left|\left(S^{n} a, b\right)\right| \leqq\left(\lim \sup \left|\left(S^{n} a, a\right)\right|\right)^{1 / 2} \cdot\|b\| .
$$


Proof. Using weak sequential compactness, we can choose $n_{k} \uparrow \infty$ such that $S^{n_{k}} a \rightarrow c$ weakly and $\lim \sup \left|\left(S^{n} a, b\right)\right|=|(c, b)|$. For each fixed $p,\left(c, S^{p} a\right)=\lim _{k}\left(S^{n k} a, S^{p} a\right)=\lim _{k}\left(S^{n_{k}-p} a, a\right)$, by successive applications of Lemma 1 . Hence $\left|\left(c, S^{p} a\right)\right| \leqq \lim \sup \left|\left(S^{n} a, a\right)\right|$, so that $\|c\|^{2}=\lim _{k}\left|\left(c, S^{n_{k}} a\right)\right| \leqq \lim \sup \left|\left(S^{n} a, a\right)\right|$. But $|(c, b)|$ $\leqq\|c\| \cdot\|b\| \cdot \square$

The following geometrical lemma seems to be new.

Lemma 3. Suppose $K$ is a closed, convex, and circled subset of $\mathfrak{H}$, and that $h \in \mathcal{H C}$ with $\|h\| \leqq 1$. For each $\alpha>0$, let $h_{\alpha}$ be the point in $\alpha K$ closest to $h$, and let $F(\alpha)=\sup \left\{\left|\left(h-h_{\alpha}, k\right)\right|: k \in \alpha K\right\}$ (thus $F(\alpha)$ measures how nearly orthogonal is the "projection" $h \rightarrow h_{\alpha}$ of h onto $\left.\alpha K\right)$. Then $F(\alpha)$ is continuous and $\int_{0}^{\infty}(F(\alpha) / \alpha) d \alpha \leqq \frac{1}{2}$.

Proof. Standard variational tricks (which we omit) show that, in fact, $F(\alpha)=\left(h-h_{\alpha}, h_{\alpha}\right)$. It is also clear that $\|h\| \leqq 1 \Rightarrow\left\|h-h_{\alpha}\right\|,\left\|h_{\alpha}\right\|$, $F(\alpha) \leqq 1$.

Set $A(\alpha)=\left\|h-h_{\alpha}\right\|^{2}$, and consider $\alpha, \beta>0$. Since $(\beta / \alpha) h_{\alpha} \in \beta K$, we have the inequality

$$
\begin{aligned}
A(\beta) & \leqq\left\|h-(\beta / \alpha) h_{\alpha}\right\|^{2}=\left\|\left(h-h_{\alpha}\right)+(1-\beta / \alpha) h_{\alpha}\right\|^{2} \\
& =A(\alpha)+(1-\beta / \alpha)^{2}\left\|h_{\alpha}\right\|^{2}+2(1-\beta / \alpha) F(\alpha) .
\end{aligned}
$$

Clearly, then, $A(\alpha)$ is a continuous function of $\alpha$. Using the "parallelogram law" and the fact that $\frac{1}{2}\left(h_{\alpha}+h_{\beta}\right) \in \frac{1}{2}(\alpha+\beta) K$, we obtain the inequality

$$
\begin{aligned}
\left\|h_{\alpha}-h_{\beta}\right\|^{2} & =2 A(\alpha)+2 A(\beta)-4\left\|h-\frac{1}{2}\left(h_{\alpha}+h_{\beta}\right)\right\|^{2} \\
& \leqq 2 A(\alpha)+2 A(\beta)-4 A\left(\frac{1}{2}(\alpha+\beta)\right) .
\end{aligned}
$$

Thus the continuity of $A(\alpha)$ implies that of $h_{\alpha}$ (as a function into $\mathcal{F}$ ), so that $F(\alpha)$ is also con tinuous.

Since $\left\|h_{\alpha}\right\| \leqq 1,(1)$ implies that

$$
A(\beta)-A(\alpha)+2(\beta-\alpha) F(\alpha) / \alpha \leqq|\beta-\alpha|^{2} / \alpha^{2} .
$$

By reversing the roles of $\alpha$ and $\beta$, we see that

$$
\begin{aligned}
& |A(\beta)-A(\alpha)+2(\beta-\alpha) F(\alpha) / \alpha| \\
& \leqq|\beta-\alpha|^{2} \cdot \max \left(\alpha^{-2}, \beta^{-2}\right)+2|\beta-\alpha| \cdot|F(\alpha) / \alpha-F(\beta) / \beta| .
\end{aligned}
$$

Since $F$ is continuous, (4) implies that $A^{\prime}(\alpha)=-2 F(\alpha) / \alpha$, and, integrating and recalling that $0 \leqq A(\alpha) \leqq 1$, we obtain the inequality of the lemma.

Now, under the conditions of Theorem 1, consider (for $\alpha>0$ ) the sets $K_{\alpha}=\left\{f \in \mathfrak{F}: \lim \sup \left|\left(T^{n} h, f\right)\right| \leqq \alpha\right\}$. Clearly each subset $K_{\alpha}$ of $\mathfrak{F}$ 
is closed, convex, and circled, and $K_{\alpha}=\alpha K$, where $K=K_{1}$. Certainly $T^{*} K_{\alpha} \subset K_{\alpha}$, and Lemma 1 (with $S=T$ ) shows that $T K_{\alpha} \subset K_{\alpha}$ also.

We define $h_{\alpha}$ and $F(\alpha)$ as in Lemma 3. If $k \in K_{\alpha}$, then also $\left(T^{*}\right)^{n} k$, $T^{n} k \in K_{\alpha}$, and hence

$$
\left|\left(T^{n} h, k\right)-\left(T^{n} h_{\alpha}, k\right)\right| \leqq F(\alpha),
$$

and

$$
\left|\left(\left(T^{*}\right)^{n} h, k\right)-\left(\left(T^{*}\right)^{n} h_{\alpha}, k\right)\right| \leqq F(\alpha) .
$$

Using (5) and the fact that $h_{\alpha} \in K_{\alpha}$, we see that $\lim \sup \left|\left(\left(T^{*}\right)^{n} h_{\alpha}, h_{\alpha}\right)\right|=\lim \sup \left|\left(T^{n} h_{\alpha}, h_{\alpha}\right)\right| \leqq \lim \sup \left|\left(T^{n} h, h_{\alpha}\right)\right|$

$$
+\lim \sup \left|\left(T^{n} h, h_{\alpha}\right)-\left(T^{n} h_{\alpha}, h_{\alpha}\right)\right| \leqq \alpha+F(\alpha) \text {. }
$$

It follows by Lemma 2 (with $a=h_{\alpha}, b=g$, and $S=T^{*}$ ) that

$$
\left.\lim \sup \mid\left(T^{*}\right)^{n} h_{\alpha}, g\right) \mid \leqq(\alpha+F(\alpha))^{1 / 2} \cdot\|g\| \text {. }
$$

Recalling the hypotheses of Theorem 1 , we see that $(\alpha / \delta) g \in K_{\alpha}$ so that, by (6),

$$
\left|\left(\left(T^{*}\right)^{n} h, g\right)-\left(\left(T^{*}\right)^{n} h_{\alpha}, g\right)\right| \leqq(\delta / \alpha) F(\alpha) .
$$

Finally, (7) and (8) yield (since $\|g\| \leqq 1$ )

$$
\lim \sup \left|\left(\left(T^{*}\right)^{n} h, g\right)\right| \leqq \delta F(\alpha) / \alpha+(\alpha+F(\alpha))^{1 / 2} .
$$

Now, if $0<\epsilon \leqq 1$, Lemma 3 ensures that we can find

$$
\alpha \in\left[\left(\epsilon^{2} / 4\right) \cdot \exp \left(-2 \epsilon^{-2}\right), \epsilon^{2} / 4\right]
$$

such that $F(\alpha) \leqq \epsilon^{2} / 4$. Then, if $\delta \leqq\left(\epsilon^{2} / 4\right) \exp \left(-2 \epsilon^{-2}\right)$, it is easy to deduce from (9) that lim sup $\left|\left(\left(T^{*}\right)^{n} h, g\right)\right| \leqq \epsilon$.

\section{REFERENCES}

1. S. R. Foguel, Powers of a contraction in Hilbert space, Pacific J. Math. 13 (1963), 551-562. MR $29 \# 473$.

2. - A counterexample to a problem of Sz.-Nagy, Proc. Amer. Math. Soc. 15 (1964), 788-790. MR 29 \#2646.

3. B. Sz.-Nagy and C. Foiaş, Analyse harmonique des opérateurs de l'espace de Hilbert, Masson, Paris; Akad. Kiadó, Budapest, 1967. MR 37 \#778.

4. P. R. Halmos, On Foguel's answer to Nagy's question, Proc. Amer. Math. Soc. 15 (1964), 791-793. MR 29 \#2647.

5. A. Zygmund, Trigonometric series. Vol. 2, 2nd ed., Cambridge Univ. Press, New York, 1959. MR 21 \#6498.

6. K. de Leeuw and Y. Katznelson, The two sides of a Fourier-Stieltjes transform and almost idempotent measures, Israel J. Math. 8 (1970), 213-229.

University of California at San Diego, la Jolla, California 92037 